\title{
Geolingüística del condicional evidencial en las lenguas romances del siglo $\mathrm{xx}$
}

\section{Geolinguistics of the Evidential Conditional in the 20th-century Romance Languages}

\author{
Víctor LARA Bermejo [victor.lara@gm.uca.es] \\ Universidad de Cádiz, España
}

\section{RESUMEN}

El condicional en gran parte de las lenguas romances expresa tiempo, modalidad y evidencialidad. Sin embargo, la bibliografía ha basado sus afirmaciones en escasos datos, en textos literarios y elevados, así como en su propia introspección. En este trabajo, pretendemos dar cuenta de la distribución geográfica y dialectal del condicional a principios del siglo xx, con el fin de determinar las lecturas semánticas que podía denotar y establecer si, como se aduce actualmente, ya era una estrategia de evidencialidad hace cien años.

\section{Palabras clave}

Condicional; geolingüística; evidencialidad; lenguas romances; atlas lingüístico

\begin{abstract}
The conditional tense in most Romance languages conveys time, modality and evidentiality. However, the authors have based their statements upon scarce data, literary texts as well as their introspection. In this article, I aim to account for the dialect and geographic distribution of the conditional tense in the early 20th century, in order to determine the semantic readings, it could express. Furthermore, I will pinpoint whether, as happens nowadays, it was already an evidentiality strategy one hundred years ago.
\end{abstract}

\section{KEYWORDS \\ Conditional tense; geolinguistics; evidentiality; Romance languages; linguistic atlas}

RECIBIDO 2021-04-03; ACEPTADO 2021-07-03

Este artículo se enmarca dentro del proyecto Evidencialidad, subjetivización y perspectivización en las interfaces de la lengua, con referencia PID2019-104405GB-100, financiado por el Ministerio de Ciencia e Innovación (España). 


\section{Introducción}

El condicional (COND) en las lenguas romances ha sido objeto de muchos estudios, debido a sus múltiples funciones semánticas. Además de expresar tiempo futuro referido a una temporalidad pretérita (es decir, es un pasado del futuro), el COND connota modalidad y, para Squartini (2001), evidencialidad en gran parte de las variedades románicas. Esta última afirmación se basa en el hecho de que, para el autor, el COND sirve para expresar inferencia referida al pasado y/o reportatividad, es decir, información que proviene de un tercero (tabla 1).

\begin{tabular}{|l|c|c|c|c|c|}
\hline & Portugués & Español & Catalán & Francés & Italiano \\
\hline Futuro & $\mathrm{I} / \mathrm{R}$ & $\mathrm{I}$ & - & $\mathrm{I}$ & $\mathrm{I}$ \\
\hline Condicional & $\mathrm{I} / \mathrm{R}$ & $\mathrm{I} /(\mathrm{R})$ & - & $\mathrm{I} / \mathrm{R}$ & $\mathrm{R}$ \\
\hline
\end{tabular}

Tabla 1: Evidencialidad en los romances ( $I=$ inferencial, $\mathrm{R}=$ reportativo)

De acuerdo con la taxonomía de Squartini (2001), el COND es inferencial referido al pasado en español, portugués y francés, así como reportativo en estos tres idiomas e italiano. Sin embargo, el autor establece que el COND reportativo en español es de creación reciente, mientras que excluye la posibilidad de reportatividad o inferencia en catalán.

Los estudios sobre el COND han girado en torno a la casuística contemporánea y se han basado sobre todo en datos cualitativos, en la introspección del investigador o en el plano estándar, pero carecemos de análisis exhaustivos que den cuenta de la realidad dialectal y del lenguaje no culto. Las primeras herramientas que tratan de recoger la situación de las variedades vernáculas romances en el ámbito europeo fueron los atlas llevados a cabo en los primeros años de 1900, de los cuales hemos extraído datos con el fin de determinar qué lecturas tenía el COND en dicho momento y hasta qué punto se puede afirmar que poseía valor evidencial.

\section{Corpus, marco teórico y metodología}

Con el fin de cartografiar la realidad del COND en todos los romances, hemos recurrido a los siguientes corpus. Para el italiano de primera mitad del siglo xx, hemos sacado los datos del Atlante linguistico ed etnográfico dell'Italia e della Svizzera meridionale (AIS). Dicho atlas se llevó a cabo entre la década de 1920 y 1940 a través de una serie de encuestas realizadas por Gerhard Rohlfs, Paul Scheuermeier y Max Leopold Wagner, las cuales se basaron en un cuestionario confeccionado por un grupo de investigadores de la Universidad de Berna. Este contenía palabras y frases preestablecidas que el informante, una persona de entorno sobre todo rural y sin movilidad que rondaba entre los 50 y los 60 (aunque se encuestaron ambos sexos, el masculino predomina), debía repetir en su variedad.

En el caso del Atlas linguistique de la France (ALF), sus creadores, Jules Gilliéron y Edmond Edmond, recorrieron entre 1897 y 1900 las 639 localidades que encuestaron en Francia, la zona valona de Bélgica y el área francófona de Suiza. Su metodología era idéntica a la del AIS. Asimis- 
mo, el Atlas lingüístico de la Península Ibérica (ALPI) se basa en el mismo método. En su caso, el atlas fue creado por Tomás Navarro Tomás y llevado a cabo por tres equipos repartidos por la zona castellana, la gallego-portuguesa y la catalano-valenciana, con dos investigadores nativos en cada grupo. Se encuestaron 527 enclaves rurales y en cada uno de ellos se entrevistó a un informante con poco nivel de estudio, que rondara entre los cuarenta y sesenta años y que toda su vida hubiera vivido en el pueblo. Las entrevistas fueron realizadas entre los años 1920 y 1950 y consistían en oraciones y palabras preconcebidas que los informantes repetían en su variedad vernácula y cuyos resultados eran manuscritos fonéticamente.

En el caso del rumano, hemos consultado el Atlasul lingvistic român (ALR), confirmando el hecho de que dicha lengua no desarrolló un COND morfológico. Aunque cuenta con una perífrasis que sirve para marcar dicho tiempo, no exhibe una forma sintética como el resto de romances. Por tanto, no ilustraremos ningún dato del rumano del siglo $\mathrm{xx}$, ya que su estrategia no coincide con la del resto de lenguas que aquí se tratan. Debemos tener en consideración la propia metodología de los atlas lingüísticos, ya que recogen una sola respuesta por informante y localidad. Empero, representan las únicas fuentes dialectales de la época. Gracias además al perfil de informante (NORM), los datos que proporcionaremos garantizarán respuestas no condicionadas por el estándar o por registros cultos.

Antes de pasar a los resultados cartográficos, queremos aclarar qué vamos a entender en este artículo por evidencial. El término se presta a muchas interpretaciones, sin que haya consenso en establecer una lectura única. En nuestro caso, seguiremos los postulados de Aikhenvald (2004, 2018), quien defiende que la evidencialidad es un sistema morfológico que connota de manera primaria, sistemática y/o única fuente de información. Este paradigma puede estar provisto mediante morfemas que se adhieren a un verbo, pero también se puede desarrollar gracias a la especialización de tiempos verbales concretos. Es esta la estrategia que más nos interesa, ya que, de acuerdo con la autora, el COND puede haberse convertido en estrategia de evidencialidad, pero solo si la lectura primaria, sistemática y/o única es la de fuente de información. Por tanto, si en una lengua dada, el COND es más temporal o modal que inferencial o reportativo, no podrá considerarse que el COND en dicho idioma sea evidencial o que la lengua posea un sistema de evidencialidad.

Para Aikhenvald $(2004,2018)$, recursos como me han dicho o aparentemente no suponen estrategias evidenciales; de lo contrario, toda lengua exhibiría evidencialidad. La autora insiste en el patrón morfológico de la evidencialidad y en su carácter primario y sistemático. Por ello, si el COND denota coherentemente inferencia o reportatividad, podremos determinar que el COND es evidencial. 


\section{Resultados}

A continuación, presentamos los resultados según el atlas dialectal consultado. Mostraremos en primer lugar los datos del ALPI, seguidos de los del ALF y, más tarde, los del AIS.

\subsection{ALPI}

El ALPI preestablecía 3 oraciones con un condicional: 386. Si tuviera dinero lo compraría; 387. Si estudiase, aprendería; 388. Si pudiera, la mataría. En primer lugar, cabe resaltar que las tres frases exhiben un COND con valor modal no conjetural. Es decir, los investigadores no previeron oraciones con un COND temporal ni tampoco inferencial o reportativo. Los resultados de conjunto se ilustran en el mapa 1.

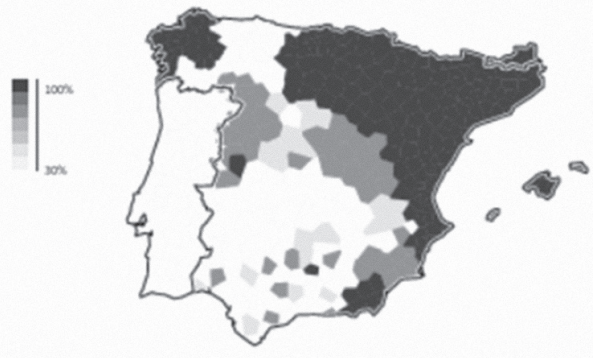

Mapa 1: COND en el ALPI

El mapa 1 indica que el COND ha sido la opción sistemática en todas las variedades de catalán y en gran parte del gallego, así como en la zona septentrional y oriental del español peninsular. Este empleo prolífico decrece en el resto de la mitad norte castellana y el extremo sureste de España, mientras que es inexistente en portugués y en gran parte del español. En estos lugares, ha sido el imperfecto de indicativo la elección preferida.

\subsection{ALF}

El ALF preveía diez oraciones con un COND: 10a. Ils s'agouinlleraient devant lui; 98. Tu aurais dî te taire; 99. Vous auriez dû voir comme les arbres en étaient chargé; 498a. Il me serrait si fort que je croyais qu'il métranglerait; 515 . Tu aurais dû te taire et nous serions loin; 516 . Tu as oublié que vous deviez nous faire signe quand vous series en haut; 537. Il faudrait être aveugle ou fou; 807. Si cétait bien cuit, j'en mengerais bien; 1366. J'ai cru qu'ils ne viendraient pas; 1419. Je voudrais bien avoir de celle-ci.

Por un lado, hay que incidir en que solo dos de las diez frases contienen un COND con valor temporal, mientras que las ocho restantes poseen un COND modal no conjetural. Es decir, ninguna formulación connotaba inferencia o reportatividad. En cualquier caso, el COND ha sido sistemático para ambas lecturas (mapa 2). 


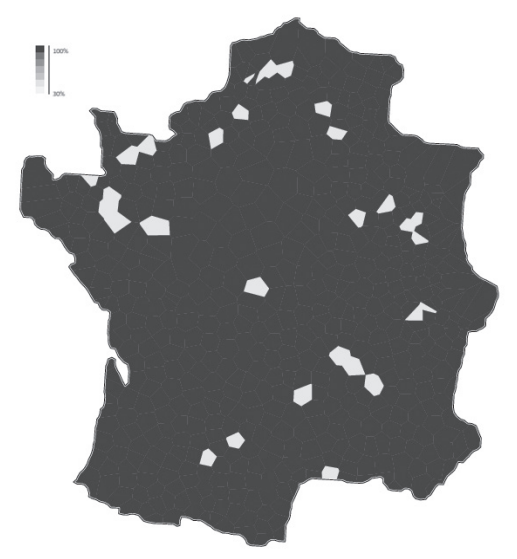

Mapa 2: COND en el ALF

El mapa 2 certifica que el COND era el recurso para un futuro referido a una temporalidad pasada, así como a un contexto modal. Al igual que sucedía con el ALPI, no hay preguntas en el ALF que contuvieran una frase con valor inferencial o reportativo con otras estrategias, como verbos modales.

\subsection{AIS}

El AIS preestablecía 14 frases con un COND: 676. Potrebbe ferirse; 1016. Mangeresti; 1019. Ne mangerei; 1035. Beverei; 1044. Comprerebbero; 1104. Mi piacerebbe; 1113. Cosa ne faresti?; 1250. Avreste dovuto vedere; 1519. Vorrei di questa qui; 1603. Vorrebbe; 1613. Lavoreremmo di più; 1627. Gli parlerei io; 1630. Non sarebbe contento; 1633. Voi lo trovereste in qualche luogo. Al igual que ocurría con el ALPI, el atlas referido al italiano no dispone de frases con un COND inferencial, reportativo o temporal, ya que las catorce oraciones presentan un matiz modal (mapa 3).

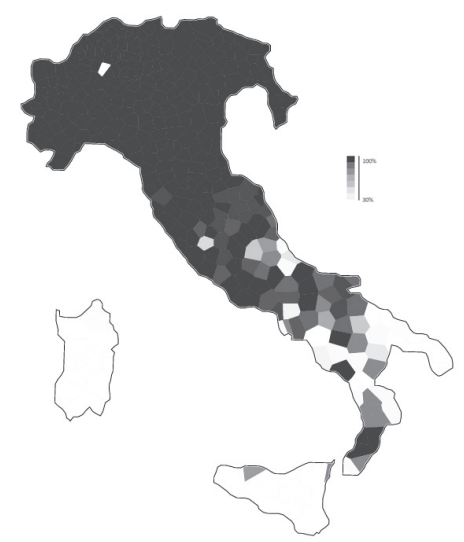

Mapa 3: COND en el AIS 
La casuística italiana expone dos realidades opuestas: una mitad norte con una sistematicidad en el uso del COND, si bien esta hegemonía es igualmente amplia en los dialectos centrales, y un extremo meridional donde el COND no aparece, lo hace de forma extraordinaria o posee enclaves que basculan hacia el comportamiento septentrional. En las islas, el COND es minoritario o inexistente, al menos para las lecturas semánticas previstas en el AIS.

\section{Análisis}

Los datos geolingüísticos expuestos en el apartado anterior sugieren que el COND a inicios del siglo pasado era, ante todo, una estrategia modal y, en menor medida, temporal. Asimismo, no podemos afirmar para dicha época que el COND fuera una estrategia de evidencialidad en ninguna de las lenguas investigadas. En primer lugar, ninguno de los atlas consultados preestablecía ninguna frase con un COND que contuviera dicha significación. Este matiz es relevante, ya que, en todos los casos, los encuestadores pretendían recoger el mayor número de fenómenos vernáculos, incluidos los morfosintácticos y no solo los fonéticos y léxicos.

Si echamos un vistazo a las obras metalingüísticas de todas las variedades, observamos que el COND sirve como modal, sobre todo para las hipótesis, y como futuro de un tiempo pasado en italiano (Rohlfs 1968). El autor indica que, en el sur, el COND tan solo sería útil para la modalidad, ya que la función temporal estaría copada por el imperfecto de indicativo, al igual que el presente de indicativo indica tiempo futuro en lugar del futuro morfológico. Asimismo, Rohlfs (1968) asegura que el COND se emplea para la reportatividad, poniendo ejemplos de lenguaje periodístico e indica que, en época medieval, se ven usos de inferencial. Ledgeway (2009) apoya la tesis de Rohlfs (1968) en cuanto al carácter menos prolífico del COND en el sur, aunque no hay referencia alguna al carácter evidencial de dicho tiempo verbal.

En cuanto al francés, Nyrop (1925) indica que el COND puede ser temporal (futuro de un pasado) y modal. El valor evidencial lo circunscribe a textos elevados del siglo xviı y época medieval, aduciendo la posibilidad inferencial. Togeby (1974) señala el valor temporal del condicional, así como modal en esta lengua, sin comentar en ningún momento cualquier matiz inferencial o reportativo. Sin embargo, otros autores apuntan a que el COND francés tenía valor evidencial ya en el siglo XVII y XVIII. Así lo establece Vatrican (2010) y Van de Weerd (2018), quienes atestiguan el COND reportativo en textos elevados, jurídicos y periodísticos.

Para las lenguas iberorromances, la bibliografía descarta el COND con valor evidencial en catalán. El español, por su parte, sí emplea en la actualidad el COND para la inferencia referida a un hecho pasado (RAE 2009) y, en el estilo periodístico, para la reportatividad, cuestión que Romero Gualda (1994) apoya, si bien acota su empleo como algo incipiente. La obra más antigua que alude al COND reportativo se encuentra en Gili Gaya (1943), quien achaca este matiz a una mala traducción del francés y el inglés. Álvarez \& Xove (2002) señalan para el gallego que, actualmente, el COND es un recurso temporal, modal y evidencial, como en español. Por último, el portugués desfavorece por completo el COND por diversos motivos: obliga a la mesoclisis cuando se añaden pronombres átonos (es decir, a la posición del clítico entre raíz y desinencia, derivando en reajustes alomórficos de acuerdo con criterios fonéticos), nunca ha sido muy prolífico históricamente, siguiendo la estela del futuro, tiende a ser sustituido por el imperfecto de indicativo y, en el caso de 
aparecer, lo hace con valor puramente evidencial, ya sea con inferencia referencia al pasado como con valores reportativos (Oliveira 1985; Cuna \& Cintra 1992).

Para entender por qué el COND de inicios del siglo xx no era evidencial y comparar si lo es en realidad a día de hoy, es necesario valorar el comportamiento del futuro morfológico y la dicotomía entre lenguaje culto y no culto. El origen y la posterior evolución del COND en las lenguas romances se han supeditado en todo momento a los avatares del futuro morfológico. Company (2006) traza el nacimiento y desarrollo de ambos tiempos verbales y corrobora que provienen de la misma perífrasis latina formada por infinitivo más HABEO, siendo el COND flexionado con el imperfecto навевам. Su evolución pasó por un proceso paulatino de gramaticalización, con la consecuente pérdida de peso fónico y su especialización en tiempo verbal. La mesoclisis que permitía al principio desapareció, aunque estos cambios no fueron simultáneos en toda el área románica, ya que el futuro y el COND evolucionaron más rápido en la zona catalana, provenzal y la Italia septentrional, pero no terminó de asentarse por completo en el occidente de la Península Ibérica, ya que, como decíamos, todavía se permite la mesoclisis y el grado de pérdida de peso fónico es mínimo en comparación con las regiones foco del fenómeno.

El comportamiento del futuro ha marcado el del COND, que ha actuado siempre por analogía, por lo que se hace necesario cotejar el funcionamiento del primero en la misma época y con datos de los mismos atlas. Lara Bermejo (en prensa) expone precisamente la distribución geolingüística del futuro morfológico, el cual surge inexorablemente para la temporalidad en francés y catalán, así como gran parte del español peninsular, el gallego y el portugués. Asimismo, las variedades italianas presentan a principios de 1900 una oposición clara entre la mitad norte, donde es prolífico para tiempo, y la mitad sur más las islas, donde no aparece para expresar posterioridad. Solo la región meridional italiana exhibía hace cien años evidencialidad en el futuro morfológico, a tenor de su inexistencia para denotar tiempo y el hecho de que Ledgeway (2009) y Rohlfs (1968) afirmen que dicho tiempo sirve para expresar inferencia. En el caso del primer autor, es el siglo xix el que marca un antes y un después en las variedades napolitanas, ya que el futuro pasa de marcar tiempo para connotar conjetura al tiempo que la temporalidad futura empieza a configurarse mediante el presente de indicativo.

El estudio de Ledgeway (2009) resulta relevante, ya que coincide con lo descubierto por Lara Bermejo (2021b) con respecto a los iberorromances. De acuerdo con el autor, la especialización del futuro morfológico como estrategia evidencial es directamente proporcional al empleo del presente de indicativo o una perífrasis para marcar tiempo futuro. Lo mismo que ocurrió en las variedades sureñas de Italia, donde el presente de indicativo empezó a connotar posterioridad y el futuro morfológico se especializó para la conjetura, ha sucedido en gran parte de la Península Ibérica. Los datos de hace cien años indican que dicho tiempo era meramente una marca de temporalidad, pero hoy en día ha revertido sus valores, ya que indica hegemónicamente conjetura, convirtiéndose en evidencial, salvo en Cataluña y Baleares. Simultáneamente a este proceso, el presente de indicativo y la perífrasis ir (a) más infinitivo ha ido adoptando los valores temporales que poseía el futuro morfológico, al igual que en los dialectos italianos del sur.

Si volvemos a los mapas sobre el COND, observamos que, en el caso de los iberorromances, es minoritario o inexistente allá donde hace cien años el futuro morfológico era también escaso. En francés, el COND es prolífico al igual que lo es el futuro morfológico en la misma época. Y en italiano, son de nuevo las islas y el extremo austral del país donde el COND no aparece, al igual 
que no surgía el futuro morfológico en los mismos años. Dicho de otra manera, hay una correlación clara entre el empleo del COND y del futuro morfológico no solo a nivel semántico, sino geolingüístico. Y al comportarse el COND por analogía del futuro morfológico, la conversión de este tiempo en evidencial es posterior a la del futuro morfológico.

Hemos anticipado que la dicotomía lenguaje culto versus no culto ha sido crucial en la generación de la evidencialidad en los romances. Las alusiones a la posibilidad de que tanto el futuro como el COND puedan generar matices evidenciales se encuentran en obras decimonónicas o en otras contemporáneas que reflejan datos de siglos pasados donde ya sea el futuro como el COND se establecen para la conjetura e incluso la reportatividad. Aaron (2014) comenta para el español que el futuro ha tenido en toda su historia el valor conjetural y el temporal. Ambos matices han convivido siempre, con una clara preferencia por el último, hasta que el siglo xx da muestras de una tendencia contraria. La autora da cuenta de esta convivencia en textos elevados, pero cuando coteja lenguaje elevado con coloquial, demuestra que todavía en 1900 es el primer caso el que da mayores ejemplos relativos de futuro conjetural frente al comportamiento del ámbito no culto, donde el valor temporal era aún la norma. Los mapas que ilustra Lara Bermejo (en prensa) corroboran los hallazgos de Aaron (2014) a este respecto.

En cuanto a otros idiomas, advertimos la misma convivencia, al menos, del futuro entre un valor temporal y otro modal que tiende al evidencial. Jensen (1990) apunta a este hecho en lo que respecta al francés, si bien todas las ocurrencias de futuro conjetural que muestra son de registros elevados. Lo mismo ocurre con el COND reportativo, ya que Van de Weerd (2018) lo circunscribe al ámbito legal y jurídico dieciochesco, tras el cual viene el periodístico, como decíamos al principio de nuestro trabajo. Sin embargo, Stage $(2002 ; 2003)$ aclara que el futuro conjetural no es admisible hoy en día en el francés estándar, aunque es aceptable si el futuro es compuesto. El italiano replica el comportamiento del francés y el español, con la convivencia temporal y modal del futuro (Rohlfs 1968), pero Squartini (2001) estudia la evolución del COND y asegura que, aunque podía ser inferencial referido al pasado, actualmente solo puede ser reportativo. No obstante, su estudio de nuevo se basa en textos elevados.

El portugués, por su parte, repite el comportamiento del italiano o el español: el aumento del presente de indicativo o la perífrasis ir más infinitivo en futuro provoca la especialización del futuro morfológico como evidencial, mientras que el empleo recurrente del imperfecto de indicativo con valor temporal de futuro del pasado incide en la especialización del COND con valor evidencial (Lara Bermejo 2021b). Por último, el catalán tiende a los valores evidenciales en el ámbito no culto, mientras que no era posible hace más de cien años; sin embargo, Martines (2017) asegura la posibilidad del valor conjetural en etapas previas, pero de nuevo los textos son de carácter erudito.

El hecho además de que en todas las lenguas el carácter reportativo del COND se achaque inexorablemente al periodismo apuntala el origen elevado de dicho valor semántico. Además, la direccionalidad en el género discursivo implica la reversión de los matices a lo largo del tiempo. Tanto el futuro como el COND surgen para expresar tiempo y valores evidenciales, pero estos últimos tan solo se dan en el plano culto y de forma minoritaria. En un momento dado, estos matices empiezan a copar también el lenguaje no elevado, el coloquial, a la vez que otras estrategias (como perífrasis o tiempos como el presente y el imperfecto de indicativo) van adquiriendo los valores temporales que el futuro y el COND van perdiendo paulatinamente. Transcurrido cierto período, la proporción de valores evidenciales del futuro y el COND aumentan al igual que los 
valores temporales de las perífrasis, el presente y el imperfecto. Posteriormente, el futuro y el COND surgen en la coloquialidad sobre todo para expresar fuente de información (conjetura y reportatividad), mientras que el valor temporal lo recogen mayoritariamente la perífrasis, el presente y el imperfecto. En última instancia, el futuro y el COND aparecen únicamente como fuente de información, mientras que la perífrasis, el presente y el imperfecto sirven sistemáticamente para marcar tiempo. Contemporáneamente a la especialización del futuro y el COND como fuente de información, estos dos tiempos se restringen como estrategias temporales en el plano culto, contraviniendo por completo el origen de todo este proceso. Así lo corroboran Escandell Vidal (2019), para el español, quien asegura que el futuro es estrategia temporal solo en el ámbito erudito; lo mismo aducen Cunha \& Cintra (1992), para el portugués o Ledgeway (2009), para las variedades meridionales de italiano.

Los resultados cartográficos no dejan lugar a dudas en torno a la falta de evidencialidad en los romances de inicios de 1900. Recordamos en este punto que la evidencialidad, tal y como la entiende Aikhenvald $(2004 ; 2018)$, consiste en un sistema morfológico que marca sistemáticamente, hegemónicamente o primariamente fuente de información, independientemente de que alguna de estas estrategias también pueda expresar otro valor gramatical, como tiempo. Los mapas expuestos indican que el COND era un recurso temporal o modal, pero en absoluto conjetural o reportativo. No solo los investigadores no previeron ninguna frase con este matiz, sino que los datos acerca del futuro morfológico corroboran esta asunción, ya que tampoco exhibía valor evidencial hace un siglo. El comportamiento del COND en las lenguas romances ha ido siempre a rebufo del futuro morfológico desde el origen de ambos tiempos; por tanto, su especialización como estrategia evidencial viene influida por la especialización previa del futuro morfológico (Lara Bermejo 2021a).

El COND podía ser reportativo e incluso inferencial hace cien años, pero esa posibilidad se constreñía al lenguaje culto, como le pasaba al futuro morfológico. La casuística actual es justamente la opuesta, pero para que se haya producido esta reversión, han hecho falta dos procesos: el primero, el traspaso de los matices evidenciales del lenguaje culto al coloquial; el segundo, la conversión gradual del COND de marca de tiempo o modalidad a marca de evidencialidad. Para Aikhenvald (2004; 2018), el futuro y el COND se prestan a ser reinterpretados como marcas de evidencialidad, ya que la incertidumbre que expresan permite dicho reanálisis. Bybee et al. (1991) apuntan de alguna manera al mismo escenario, puesto que indican que, interlingüísticamente, el futuro suele transitar por una serie de fases, como se especifica a continuación: posesión > obligación > intención > futuro > conjetura e imperativo. El futuro morfológico en las lenguas romances se inició con valor de obligación, hasta que se reanalizó como estrategia de tiempo futuro. Actualmente, se concibe como valor conjetural en gran parte de los romances, al menos en el ámbito no culto, siendo por tanto evidencial. La importancia que otorgamos al futuro morfológico se debe a que el COND romance representa exactamente la misma estrategia, aunque con valor pretérito. Tanto es así, que el tipo de conjetura expresada es aquella referida al pasado y, en el caso de la reportatividad, aquella información cuya fuente es más lejana que la connotada por otros medios.

Para que el COND pueda considerarse evidencial, aunque exprese conjetura y/o reportatividad, es necesario que la lectura conjetural y/o reportativa sea la principal, la sistemática o la primaria. Si es secundaria, nos hallamos simplemente ante una extensión semántica más de dicho tiempo verbal. Es, no obstante, el preludio de la evidencialidad, tal y como comenta Aikhenvald (2004; 2018), ya que existen muchos tiempos verbales que pueden llegar a expresar fuente de información. 
No obstante, esta posibilidad no los confiere de valor evidencial, pero inicia una fase que puede acabar en la creación de un sistema de evidencialidad a través del empleo sistemático de dichos tiempos verbales para expresar fuente de información. Es el caso actual del futuro morfológico y el COND en gran parte de los iberorromances (Lara Bermejo 2021a), pero no lo era a principios de la centuria de 1900, ya que el COND (y el futuro morfológico) era sobre todo un recurso temporal o modal, pero no evidencial.

La estrecha relación en los romances entre futuro y COND es tal que el tipo de evidencialidad desarrollada por el último concuerda con su origen y valor temporal. En primer lugar, si denota inferencia, esta es siempre referida a un evento del pasado, mientras que el futuro la expresa para un evento del presente o de un marco temporal posterior. Si connota reportatividad, puede funcionar de dos maneras: o como valor complementario al futuro conjetural, o conviviendo con el futuro también para la reportatividad. Esta última posibilidad se da en portugués y la diferencia estriba en que el futuro marca una información con una fuente más fiable que la que se infiere del COND. La primera opción demuestra de nuevo la distribución temporal entre futuro y COND que se materializa en otros matices, ya que este último se especializa para fuente de información que proviene de un tercero, es decir, de una fuente más lejana, menos fiable, que la que otorga el futuro, el cual suele expresar conjetura, es decir, fuente de información que proviene del mismo hablante. La distancia temporal caracterizada por el pretérito que se incluye en el COND y sus valores hipotéticos favorecen su reinterpretación como estrategia evidencial que expresa fuente de información más indirecta que el futuro morfológico.

\section{Conclusiones}

El COND hace cien años marcaba tiempo (futuro del pasado) y modalidad (sobre todo, hipotética), pero el ámbito coloquial desconocía o apenas presenciaba el empleo de dicho tiempo con valor ya sea conjetural como reportativo. Sí lo hacía el plano culto, tal y como acontecía con el futuro morfológico. Los estudios indican la convivencia del COND (y el futuro) con valor temporal, modal e incluso evidencial a lo largo de los siglos, pero esta última lectura se ha acotado al discurso elevado, hasta que, a cierta altura, el recurso evidencial salta al ámbito coloquial a la vez que el valor temporal empieza a ser expresado mediante alguna perífrasis o el presente e imperfecto de indicativo. Con el tiempo, el valor evidencial se especializa como el matiz primario, asentándose como marca morfológica de evidencialidad, y la lectura temporal u otra modal se configura a través de otras estrategias.

El COND romance no era evidencial hace un siglo, entre otras cosas, porque el futuro morfológico tampoco lo era o acababa de convertirse en tal en ciertas variedades. La evolución de ambos tiempos ha sido pareja desde sus inicios, siendo el COND el que actuaba por analogía. Desde el punto de vista geolingüístico, el COND temporal y modal era más prolífico en las mismas zonas donde el futuro morfológico era el recurso más frecuente para los mismos matices: el área catalana, la zona francófona (sobre todo, provenzal y occitana), así como el norte de Italia. Sin embargo, decaía en gran parte del español, el occidente iberorromance, así como la mitad sur de Italia, Cerdeña y Sicilia. 
La evolución del COND, a tenor de las investigaciones dialectales, señala su especialización ya sea para la inferencia referida al pasado y, por tanto, en consonancia con su lectura temporal pretérita, como para la reportatividad. Esta elección no parece fortuita, puesto que la fuente de información reportativa procede de un tercero, su fiabilidad es menor, se asocia con un origen más lejano que la inferencia o la conjetura y, por tanto, obedece a la semántica que le confiere ser un tiempo referido a un evento pretérito o hipotético. No obstante, su reanálisis como marca morfológica de evidencialidad solo es posible después de que el futuro haya hecho lo propio, convirtiéndose, por su parte, en fuente de información más cercana, inmediata y fiable: el propio hablante.

\section{Referencias bilbiográficas}

Aaron, J. E. (2014). A certain future: epistemicity, prediction, and assertion in Iberian Spanish future expression. Studies in Hispanic and Lusophone Linguistics, 7, 2, 215-240.

Aikhenvald, A. (2004). Evidentiality. Oxford: Oxford University Press. . (2018). The Oxford handbook of evidentiality. Oxford: Oxford University Press.

Álvarez, R.; \& Xove, X. (2002). Gramática da lingua galega. Vigo: Galaxia.

[AIS]: Jaberg, K.; \& Jud, J. (1928-1940). Atlante linguistico ed etnografico dell'Italia e della Svizzera meridionale. Milán: Unicopli.

[ALF]: Guilliéron, J.; \& Edmont, E. (1902-1910). Atlas linguistique de la France. París: Champion.

[ALR]: Pop, S.; \& Petrovici, E. (1930-1938). Atlasul lingvistic român. Cluj Napoca: Academiei Române.

Bybee, J. et al. (1991). Back to the future. In E. C. Traugott, \& B. Heine (Eds.), Approaches to grammaticalization (pp. 17-58). Ámsterdam: John Benjamins.

Company, C. (2006). Los futuros y condicionales. In C. Company (Ed.), Sintaxis histórica de la lengua española (pp. 349-422). Ciudad de México: UNAM / Fondo de Cultura Económica.

Cunha, C.; \& Cintra, L. F. L. (1992). Nova gramática do português contemporâneo. Lisboa: João Sá de Costa.

Escandell Vidal, V. (2019). El futuro simple del español. Sistema natural frente a usos cultivados. Verba Hispanica, 26, 17-35.

Gili Gaya, S. (1943). Curso superior de sintaxis española. Barcelona: Vox.

Jensen, F. (1990). Old French and comparative Gallo-Romance syntax. Tübingen: Niemeyer.

Lara Bermejo, V. (2021a). Futuro y condicional en las lenguas iberorromances: de marca de tiempo a marca de evidencialidad. Borealis, 10, 1, 145-162.

- (2021b). El futuro morfológico en las lenguas iberorromances: temporalidad, modalidad y evidencialidad. Revue Romane, 56, 2, 297-326.

- (en prensa). Geolingüística del futuro evidencial en las lenguas romances a principios del siglo XX. Boletín de la Academia Peruana de la Lengua.

Ledgeway, A. (2009). Grammatica diacronica del napoletano. Tübingen: Max Niemeyer.

Martines, J. (2017). Lémergence des futurs épistémiques romans. L’exemple du catalanmédiéval du XIIIème siècle. En L. Baranzini (ed.), Le futur dans les langues romanes (pp. 133-168). Berna: Peter Lang.

Nyrop, K. (1925). Grammaire historique de la langue française. Copenhague: Nordisk Forlag.

Oliveira, F. (1985). O futuro em português: alguns aspectos temporais e/ou modais. In Actas do $1^{\circ}$ Encontro da Associação Portuguesa de Lingüística (pp. 353-373). Lisboa: Associação Portuguesa de Linguística. 
Real Academia Española. (2009). Nueva gramática de la lengua española. Madrid: Espasa.

Rohlfs, G. (1968). Grammatica storica della lingua italiana e dei suoi dialetti. Turín: Einaudi.

Romero Gualda, M. V. (1994). El español hablado en los medios de comunicación. Madrid: Arco Libros.

Squartini, M. (2001). The internal structure of evidentiality in Romance. Studies in Language, 25, 2, 297-334.

Stage, L. (2002). Les modalités épistémique et déontique dans les énoncés au futur (simple et composé). Revue Romane, 37, 44-66.

Stage, L. (2003). Les valeurs modales du futur et du présent. In B. Birkelund et al. (eds.), Aspects de la modalité (pp. 203-216). Tübingen: Max Niemeyer.

Togeby, K. (1974). Précis historique de grammaire française. Copenhague: Akademisk Forlag.

Van de Weerd, J. (2018). Vers les origines sémantiques du conditionnel épistémique. Étude d'un genre juridique en français classique (XVIe-XVIIIe siècles). Langue Française, 4, 77-89.

Vatrican, A. (2010). La modalité et le conditionnel de rumeur en français et en espagnol. Modèles Linguistiques, 31, 62, 83-94. 